\title{
Integration Challenge of Silicon Photonics with Microelectronics
}

\author{
Mario Paniccia, Ansheng Liu \\ Photonics Technology Lab, Intel Corporation \\ 2200 Mission College Blvd, Santa Clara, CA 95054, USA \\ Nahum Izhaky, Assia Barkai \\ Intel Corporation \\ S.B.I Park Har Hotzvim, Jerusalem, 91031, Israel
}

\begin{abstract}
In this presentation we discuss some practical issues and challenges associated with processing silicon photonic devices in a high volume CMOS manufacturing environment and the challenges of integrating the photonic devices with microelectronics.
\end{abstract}

\section{Introduction}

In the past year, there have been significant progress and performance breakthroughs in the field of silicon photonics. Fast silicon optical modulator [1, 2] and Raman silicon laser [3] based on Siliconon-Insulator (SOI) have been demonstrated. Such an advance in the development of individual silicon photonic devices boosts the promise that silicon could in the near future be considered as an optical material for integrated photonic circuits. If an integrated silicon photonic chip is developed, it could bring significant, even revolutionary, changes to the next generation communications industry by radically altering the price, power, and size for these integrated photonic components. However, significant technical challenge exists for monolithic integration of photonic and electronic circuits. A complex set of considerations should be carefully examined in regards to which application will have a better chance of success using silicon based integrated product solution. Among these considerations are the existing fab equipment capabilities, the processing restrictions associated with current process flows, the process generation (typically referred to as lithography node generation), and packaging requirements. In this paper, we will discuss some of these technical challenges associated with photonics and electronics integration.

\section{Photonics and electronics integration challenges}

Photonic and electronic integration can be divided into two categories. One may be integrating multiple photonic devices on a single chip with electronics hybrid integrated. Such an integrated photonic chip provides multiple functionalities with possibly smaller footprints as compared to discrete components based optical systems. Another would be monolithic integration of various photonic devices and electronics on a single platform. The hybrid integration may provide high performance of the integrated photonic circuits because photonic and electronic devices can be made of different material systems and each of them could be optimized for high performance. However, the key benefit of the monolithic integration would be the cost reduction, since packaging related cost of individual photonic components is largely eliminated and a large number of photonic and electronic devices could be processed in a cost effective manner on a single substrate, taking advantage of the potential of high volume manufacturing. The key challenges and issues include integration platform choice while keeping on high performance, process compatibility, yield, and packaging. 


\section{Silicon platform}

The material choice for monolithic integration is one of the major issues. Such a material must provide a common platform for both electronics and photonics. It is a well known fact that silicon is the mainstay electronics material. The question is if silicon can be used as a photonic material. In the wavelength range of $1270-1625 \mathrm{~nm}$, silicon is essentially transparent. It is therefore possible to be used to guide light. In fact, the large index contrast $(\sim 2)$ of silicon/oxide waveguide system makes it favorable to fabricate high density photonic devices. Most Optical materials used in integrated optics provide index contrast of 1-4 orders of magnitude lower, hence Si photonics allows reducing the minimal bend radius from millimeters/centimeters to micrometers, which in turn provides about 3 orders of magnitude higher area condensation! Silicon-on-insulator with rather low cost per unit of area and various EPI layer thickness and doping levels provides an ideal platform to fabricate both passive and active devices such as filters and fast modulators [1, 2]. However, no absorption at the telecom wavelength range $(1.27$ to $1.62 \mu \mathrm{m})$ poses a challenge for achieving efficient detection at these wavelengths. Introducing SiGe alloys is an established method to stretch the absorption edge to $1550 \mathrm{~nm}$. The use of high germanium concentration of strained films may compromise the material if high temperatures $(\sim>700 \mathrm{C})$ are applied. This is due to the introduction of dislocations, so special attention should be made to the implications of SiGe integration. Most leading CMOS IC manufacturing processes rely on engineered EPI-Si layer, therefore, in the case of optical and electrical monolithic integration, using SOI may mean a tweaking of the transistor processing setup to keep its designed performance. Another challenge using silicon as a monolithic integration platform is lack of silicon based laser. More research effort is needed in that direction.

\section{Process compatibility}

Realizing CMOS compatible monolithic integration will require the development of a process flow that combines the electrical and the optical sections while avoiding many potential roadblocks. Although each part of the process may be feasible in the same environment, unwanted interaction of processing steps may result in performance degradation and, in extreme cases, complete loss of functionality. Some examples of these interactions may be driven through thermal "history", wafer topography and chemical contamination. A considerable effort should be made to optimize the process flow to minimize these effects but compensation through processing setup may be needed as well. Thermal crosstalk between active (including electronics) and other active/passive elements is a significant parameter to fully consider via design or process solutions. Otherwise severe performance degradation may occur.

Traditional electrical processing is commonly separated into two parts: (a) front-end: formation of the transistor where the use of high temperatures $(>900 \mathrm{C})$ is common for gate oxide and implant diffusions. In addition, metallic contamination is extremely low since it poses a high risk. For the back-end formation of interconnects, typically limit the temperature of the wafer to below $450 \mathrm{C}$. Similar determination of front-end and back-end sections should be made for the hosted optical process. Clearly any high temperature step, such as oxidation and doping activation, must be a frontend step; even so, such a step could result in the inter-diffusion of already implanted dopant species, thus degrading transistor performance. The opposite effect, degradation of an optical device, may happen if the process order is swapped. Introducing strained SiGe may create an even more difficult restriction since as it is done in a rather high temperature but may lose its high crystalline quality if heated again.

Yet another set of design rules is related to the topography of the wafer. As wafer planarity is needed for modern photolithography, Chemical Mechanical Polishing (CMP) is often used. As a 
result, one would have to set process steps order in a way that CMP could be applied without topography conflict. CMP short-scale uniformity is very sensitive to the feature density. Therefore, masking of electronic processes will often include dummy structures to reduce density fluctuation on the scale of several micrometers. Such methodologies could prove difficult for optical mask design since they may require large masked or exposed areas, such as detector active region.

\section{Yield}

Over decades of CMOS development, automated testing of devices at the wafer level has been instrumental in reducing per-wafer manufacturing costs. Mature in-line defect metrology equipment and methodologies have been instrumental to increasing yield and providing stable device performance. The integrated process will only be advantageous if one can place strict controls on equipment cleanliness as well as easy to implement detection. As with any other process, there will be new types of defect mechanisms and interactions that will need to be understood and eliminated. On the electrical parametric front, there are at present no convenient, fast, automated, and nondestructive optical probing techniques that can be well correlated to the final, packaged optical device performance. Some efforts have been made to use top-down probing of optical devices (e.g. prism, grating, and evanescent couplers), but none of these techniques have been successfully introduced into the manufacturing process.

\section{Packaging}

Back-end processing of the integrated device will need to incorporate preparation for the attachment of fiber and possibly also an external light source. Coupling light into and out of a silicon chip is very challenging. Particularly difficult is the coupling of light from a standard single mode optical fiber to a silicon waveguide due to the large modal mismatch. Few types of tapering designs have been developed in an effort to reduce the optical loss at each transition onto or off of the optical chip. Each design poses a unique etch-process challenge to meet its requirements. As waveguide dimensions are reduced $(\sim 1 \mathrm{um})$ for performance, process challenges become more difficult (roughness and CD control over non planar wafer topography). As III-V lasers will most likely be required as on-chip light sources, passive alignment is an attractive solution, as it exploits silicon low cost and high resolution lithography to perform the alignment. The silicon substrate could be used to increase placement accuracy by using mechanical etch stops. The required placement accuracy of 1-2 $\mu \mathrm{m}$ may be satisfied by commercial pick-and-place tools, and may accommodate high volume capacity. In addition, accurate control of the etch depth is required to ensure that the vertical alignment can also meet the comparable alignment tolerance.

\section{Conclusion}

Significant progress has been made in silicon-based optoelectronic devices over the last decade, but the integration of many optical devices or optical and electrical devices is required to fully achieve the benefits of this technology. Several challenges for integration into a CMOS process have been outlined. By understanding these and similar issues, the integration of optical devices in silicon may someday become a reality.

\section{References}

[1] A. Liu et. al. "A high-speed silicon optical modulator based on a metal-oxide-semiconductor capacitor," Nature 427, 615 (2004).

[2] L. Liao et. al. "High-speed silicon Mach-Zehnder modulator," Opt. Express 13, 3129 (2005).

[3] H. Rong et. al. “A continuous-wave Raman silicon laser,” Nature 433, 725 (2005). 